\title{
TRIGONOMETRIC DARBOUX TRANSFORMATIONS AND CALOGERO-MOSER MATRICES
}

\author{
LUC HAINE \\ Department of Mathematics, Université catholique de Louvain, Chemin du Cyclotron 2, \\ 1348 Louvain-la-Neuve, Belgium \\ e-mail:luc.haine@uclouvain.be \\ EMIL HOROZOV \\ Department of Mathematics and Informatics, Sofia University, 5 J. Bourchier Boulevard, \\ Sofia 1126, Bulgaria and \\ Institute of Mathematics and Informatics, Bulgarian Academy of Sciences, Acad. G. Bonchev Str., \\ Block 8, 1113 Sofia, Bulgaria \\ e-mail:horozov@fmi.uni-sofia.bg \\ and PLAMEN ILIEV \\ School of Mathematics, Georgia Institute of Technology, Atlanta, GA 30332-0160, USA \\ e-mail:iliev@math.gatech.edu
}

\begin{abstract}
We characterize in terms of Darboux transformations the spaces in the Segal-Wilson rational Grassmannian, which lead to commutative rings of differential operators having coefficients which are rational functions of $e^{x}$. The resulting subgrassmannian is parametrized in terms of trigonometric Calogero-Moser matrices.
\end{abstract}

2000 Mathematics Subject Classification. 35Q53, 37K10

1. Introduction. The rational Segal-Wilson Grassmannian $\mathrm{Gr}^{r a t}$ parametrizes the soliton solutions of the Kadomtsev-Petviashvili (KP) equation. In [10], Wilson embarked upon a study of a subgrassmannian $G r^{a d} \subset G r^{r a t}$ that he called the adelic Grassmannian, which parametrizes the solutions of the KP equation, rational in $x$ and vanishing as $x \rightarrow \infty$. The adelic Grassmannian has (and is indeed characterized by) a remarkable bispectral involution $V \rightarrow b(V), V \in G r^{a d}$, which exchanges the role of the 'space' and the 'spectral' variables in the corresponding stationary wave functions $\psi_{V}(x, z)$, that is

$$
\psi_{b(V)}(x, z)=\psi_{V}(z, x) .
$$

In particular, the reduced stationary wave function $e^{-x z} \psi_{V}(x, z)$ depends rationally not only on $z$, but also on $x$. In [11], Wilson gave an illuminating explanation for this involution, by showing that

$$
\psi_{V}(x, z)=e^{x z} \operatorname{det}\left\{I-(x I-X)^{-1}(z I-Z)^{-1}\right\},
$$

with $(X, Z)$ an element of a so-called Calogero-Moser space

$$
C_{N}=\{(X, Z) \in g l(N, \mathbb{C}) \times g l(N, \mathbb{C}): \operatorname{rank}([X, Z]+I)=1\} / G L(N, \mathbb{C}) .
$$


Here, $g l(N, \mathbb{C})$ denotes the space of complex $N \times N$ matrices, $I$ is the identity matrix and the complex linear group $G L(N, \mathbb{C})$ acts by simultaneous conjugation of $X$ and $Z$. The bispectral involution (1.1) becomes transparent when expressed at the level of Calogero-Moser spaces, as it is given by $(X, Z) \rightarrow\left(Z^{t}, X^{t}\right)$, where $X^{t}$ and $Z^{t}$ are the transposes of $X$ and $Z$.

In [5], one of us, motivated by previous studies of the first and third authors on a discrete-continuous version of the bispectral problem in [6] and [7], suggested to study yet another subgrassmannian of $\mathrm{Gr}^{\text {rat }}$, the so-called trigonometric Grassmannian $G r^{\text {trig }} \subset G r^{r a t}$, characterized by the property that the reduced stationary wave function $e^{-x z} \psi_{V}(x, z)$ should depend rationally on $e^{x}$. The aim of this paper is to establish a formula similar to (1.2), for a space $V \in G r^{\text {trig }}$, namely

$$
\psi_{V}(x, z)=e^{x z} \operatorname{det}\left\{I-X\left(e^{x} I-X\right)^{-1}(z I-Z)^{-1}\right\},
$$

with $(X, Z)$ belonging now to a trigonometric Calogero-Moser space

$$
\begin{aligned}
C_{N}^{\text {trig }}=\{(X, Z) \in G L(N, \mathbb{C}) \times g l(N, \mathbb{C}): \\
\left.\quad \operatorname{rank}\left(X Z X^{-1}-Z+I\right)=1\right\} / G L(N, \mathbb{C}) .
\end{aligned}
$$

The formula was conjectured in [5] and established only for a generic situation. There are two main ingredients in the proof, which will allow us to derive the result from (1.2). First, the notion of bispectral Darboux transformations as introduced and used in other contexts by the second author in collaboration with Bakalov and Yakimov in [1] and [2]; second, the discovery in [6] that if $\tau\left(t_{1}, t_{2}, t_{3}, \ldots\right)$ is a tau-function of the KP hierarchy, then

$$
\tau\left(n, t_{1}, t_{2}, t_{3}, \ldots\right)=\tau\left(t_{1}+n, t_{2}-\frac{n}{2}, t_{3}+\frac{n}{3}, \ldots\right), \quad n \in \mathbb{Z}
$$

is a tau-function of the discrete KP hierarchy. Indeed, the trick to establish (1.3) is to show via the technique of bispectral Darboux transformations, that

$$
\psi_{V}^{b}(n, z)=\psi_{V}(\log (1+z), n)
$$

is a (stationary) wave function of the discrete KP hierarchy, which can be constructed from an adelic tau-function $\tau_{b(V)}\left(t_{1}, t_{2}, \ldots\right)$, with $b(V) \in G r^{a d}$, via the formula (1.5).

Finally, we like to mention that during the ISLAND 3 conference where this work was presented, Oleg Chalykh and Alexander Varchenko informed us that they have obtained results related to ours, though with rather different aims and techniques (O. A. Chalykh, private communication, July 2007; see also [9]).

2. The rational Grassmannian. In this section, we review the definition of the rational Grassmannian $G r^{r a t}$ in terms of Darboux transformations, following [1].

Definition 2.1. A function $\psi(x, z)$ is a Darboux transform of $e^{x z}$, if and only if there exist monic polynomials $f(z), g(z)$ and monic differential operators 
$P(x, \partial), Q(x, \partial)$, with $\partial=\frac{\partial}{\partial x}$, such that

$$
\begin{aligned}
\psi(x, z) & =\frac{1}{f(z)} P(x, \partial) e^{x z} \\
e^{x z} & =\frac{1}{g(z)} Q(x, \partial) \psi(x, z),
\end{aligned}
$$

with the order of $P(x, \partial)$ equal to the degree of $f(z)$.

Obviously

$$
Q(x, \partial) P(x, \partial) e^{x z}=f(z) g(z) e^{x z},
$$

so that denoting the polynomial $f(z) g(z)$ by $h(z)$, we see that

$$
h(\partial)=Q(x, \partial) P(x, \partial) .
$$

On the other hand, $\psi(x, z)$ satisfies

$$
P(x, \partial) Q(x, \partial) \psi(x, z)=f(z) g(z) \psi(x, z),
$$

showing that the operator $L=P Q$ is a traditional Darboux transformation of the operator $h(\partial)$ with constant coefficients, which justifies the terminology.

We shall denote by $\mathbb{C}[z]$ (resp. $\mathbb{C}(z)$ ) the space of polynomials (resp. rational functions) in $z$. According to [10], the rational Grassmannian $G r^{r a t}$ is formed by subspaces $V \subset \mathbb{C}(z)$ satisfying

$$
g(z) \mathbb{C}[z] \subset V \subset f(z)^{-1} \mathbb{C}[z]
$$

where $f(z)$ and $g(z)$ are polynomials, and the codimension of $V$ in $f(z)^{-1} \mathbb{C}[z]$ is equal to the degree of $f(z)$. Using the terminology of Sato's theory of the KP equation, the stationary wave function $\psi_{V}(x, z)$ (the Baker function in [10]) of such a space turns out to be a Darboux transform of $e^{x z}$, as defined earlier. In fact, the two notions are equivalent. We shall need the bilinear form on the space $\mathbb{C}(z)$ defined by

$$
B(u, v)=\operatorname{res}_{z} u(z) v(z), \quad u, v \in \mathbb{C}(z),
$$

with $\operatorname{res}_{z}$ the coefficient of $z^{-1}$ in the Laurent expansion around $\infty$.

Proposition 2.2. A function $\psi(x, z)$ is the stationary wave function of a space $V \in G r^{r a t}$ if and only if it is a Darboux transform of $e^{x z}$.

Proof. We only sketch the proof of the 'if' part. The operator $P$ in (2.1) being monic is given by

$$
P(\phi)=\frac{W r\left(\phi_{1}, \ldots, \phi_{K}, \phi\right)}{W r\left(\phi_{1}, \ldots, \phi_{K}\right)},
$$

with $\phi_{1}, \ldots, \phi_{K}$, a basis of the kernel of $P$, where $W r$ denotes the Wronski determinant. From the factorization (2.3), we have that $\operatorname{ker} P \subset \operatorname{ker} h(\partial)$ and $\operatorname{ker} Q=P(\operatorname{ker} h(\partial))$. Since $h(\partial)$ is a constant coefficients operator, it follows that the coefficients of $P$ and $Q$ are rational functions of $x$ and $e^{\lambda x}$, for a finite number of values of $\lambda$. 
Hence, if we introduce the function

$$
\psi^{*}(x, z)=\frac{1}{g(z)} Q^{*} e^{-x z},
$$

with $Q^{*}$ the formal adjoint of $Q$, defined by $(a(x) \partial)^{*}=-\partial \circ a(x)$, both $e^{-x z} \psi(x, z)$ and $e^{x z} \psi^{*}(x, z)$ are rational functions of $x, e^{\lambda x}$ and $z$. Assuming (without loss of generality) that these functions are regular at $x=0$, the coefficients of the Taylor expansions of $\psi(x, z)$ and $\psi^{*}(x, z)$ around $x=0$, generate two subspaces $V$ and $V^{*}$ of $\mathbb{C}(z)$, such that

$$
\begin{aligned}
V & =\operatorname{span}\left\{\partial^{i} \psi(x, z)_{\mid x=0}, \quad i=0,1,2, \ldots\right\} \subset f(z)^{-1} \mathbb{C}[z], \\
V^{*} & =\operatorname{span}\left\{\partial^{i} \psi^{*}(x, z)_{\mid x=0}, \quad i=0,1,2, \ldots\right\} \subset g(z)^{-1} \mathbb{C}[z],
\end{aligned}
$$

and the codimension of $V$ in $f(z)^{-1} \mathbb{C}[z]$ is equal to the degree of $f$.

The spaces $V$ and $V^{*}$ are orthogonal with respect to the bilinear form $B$ defined in (2.6), as follows from a simple computation in the ring of formal pseudo-differential operators. Indeed from (2.1) and (2.8),

$$
\psi(x, z)=P \circ f(\partial)^{-1} e^{x z} \text { and } \psi^{*}(x, z)=\left(g(\partial)^{-1} \circ Q\right)^{*} e^{-x z} .
$$

Hence, for all $i, j \geq 0$, by the 'very simple and extremely useful lemma' 6.2 .5 of [4], using (2.3), we compute

$$
\begin{aligned}
& \operatorname{res}_{z} \partial^{i} \psi(x, z) \partial^{j} \psi^{*}(x, z) \\
& \quad=(-1)^{j} \operatorname{res}_{z}\left(\partial^{i} \circ P \circ f(\partial)^{-1} e^{x z}\right)\left(\left(g(\partial)^{-1} \circ Q \circ \partial^{j}\right)^{*} e^{-x z}\right) \\
& \quad=(-1)^{j} \operatorname{res}_{\partial}\left(\partial^{i} \circ P \circ f(\partial)^{-1}\right) \circ\left(g(\partial)^{-1} \circ Q \circ \partial^{j}\right) \\
& \quad=(-1)^{j} \operatorname{res}_{\partial} \partial^{i} \circ P \circ(Q \circ P)^{-1} \circ Q \circ \partial^{j} \\
& \quad=(-1)^{j} \operatorname{res}_{\partial} \partial^{i+j}=0,
\end{aligned}
$$

with res $_{\partial} \sum a_{k} \partial^{k}=a_{-1}$. Taking the orthogonal complement with respect to $B$ of (2.10), we deduce that $g(z) \mathbb{C}[z] \subset V$, which on combining with (2.9), establishes that $V \in G r^{r a t}$, as defined in (2.5). It is easy to check that $\psi(x, z)=\psi_{V}(x, z)$, which finishes the proof.

\section{The trigonometric Grassmannian.}

DEFINITION 3.1. A Darboux transform $\psi(x, z)$ of $e^{x z}$, will be called trigonometric if and only if the operators $P$ and $Q$ in (2.1) and (2.2) have coefficients which are rational functions of $e^{x}$, i.e. $P, Q \in \mathbb{C}\left(e^{x}\right)[\partial]$.

In this section, we characterize the trigonometric Darboux transforms. With the notations of the previous section, let us write the constant coefficients operator $h(\partial)$ in (2.3) as

$$
h(\partial)=\prod_{r=1}^{n} \prod_{j=0}^{n_{r}}\left(\partial-\lambda_{r}+j\right)^{m_{r \cdot j}},
$$

where $\lambda_{1}, \ldots, \lambda_{n}$ are distinct complex numbers such that $\lambda_{r}-\lambda_{s} \notin \mathbb{Z}$ for $r \neq s$ and $m_{r, j}$, the multiplicities of the roots $\lambda_{r}-j$, are non-negative integers, with $m_{r, 0}>0$. Then, the 
kernel of $h(\partial)$ is given by

$$
\operatorname{ker} h(\partial)=\bigoplus_{r=1}^{n} W_{r}
$$

where

$$
W_{r}=\operatorname{span}\left\{x^{k} e^{\left(\lambda_{r}-j\right) x}: k=0,1, \ldots, m_{r, j}-1, j=0,1, \ldots, n_{r}\right\} .
$$

Lemma 3.2. Let $P \in \mathbb{C}\left(e^{x}\right)[\partial]$ be an operator such that the factorization (2.3) holds. Then

(i) $\phi(x) \in \operatorname{ker} P \Rightarrow \phi(x+2 \pi i l) \in \operatorname{ker} P, \forall l \in \mathbb{Z}$.

(ii) ker $P=\bigoplus_{r=1}^{n}\left(W_{r} \cap \operatorname{ker} P\right)$.

Proof. (i) The assertion follows immediately from the invariance of the coefficients of $P$ under the change $x \rightarrow x+2 \pi i l, l \in \mathbb{Z}$, since they are rational functions of $e^{x}$.

(ii) Since ker $P \subset \operatorname{ker} h(\partial)$, any $\phi(x)$ in the kernel of $P$ can be expanded as

$$
\phi(x)=\sum_{r=1}^{n}\left\{\sum_{k=0}^{k_{r}} p_{r, k}\left(e^{-x}\right) x^{k}\right\} e^{\lambda_{r} x},
$$

with $p_{r, k}\left(e^{-x}\right) \in \mathbb{C}\left[e^{-x}\right]$, some polynomial in $e^{-x}$. The result will follow by induction, if we can show that for every $r \in\{1,2, \ldots, n\}$, there exists an element $\hat{\phi}_{r}(x) \in \operatorname{ker} P$ of the form

$$
\hat{\phi}_{r}(x)=\left\{p_{r, k_{r}}\left(e^{-x}\right) x^{k_{r}}+\sum_{k<k_{r}} \hat{p}_{r, k}\left(e^{-x}\right) x^{k}\right\} e^{\lambda_{r} x},
$$

with the same polynomial $p_{r, k_{r}}\left(e^{-x}\right)$ in front of the highest power $x^{k_{r}}$ and some other polynomials $\hat{p}_{r, k}\left(e^{-x}\right)$ as coefficients of $x^{k}, k<k_{r}$.

To establish the existence of $\hat{\phi}_{r}(x)$, we observe that since $\phi(x+2 \pi i) \in \operatorname{ker} P$, for any $s \in\{1,2, \ldots, n\}$, we have that

$$
\begin{aligned}
\bar{\phi}(x) & =\phi(x)-e^{-2 \pi i \lambda_{s}} \phi(x+2 \pi i) \\
& =\sum_{r=1}^{n}\left\{\left(1-e^{2 \pi i\left(\lambda_{r}-\lambda_{s}\right)}\right) p_{r, k_{r}}\left(e^{-x}\right) x^{k_{r}}+\sum_{k<k_{r}} \bar{p}_{r, k}\left(e^{-x}\right) x^{k}\right\} e^{\lambda_{r} x}
\end{aligned}
$$

is an element of the kernel of $P$. Since $\lambda_{r}-\lambda_{s} \notin \mathbb{Z}$, for $r \neq s$, the coefficient of $x^{k_{r}}$ is the same polynomial $p_{r, k_{r}}\left(e^{-x}\right)$ multiplied by a non-zero constant for $r \neq s$ and vanishes for $r=s$. Iterating the process, we can produce an element from the kernel of the form

$$
\sum_{r \neq s}\left\{c_{r, k_{r}} p_{r, k_{r}}\left(e^{-x}\right) x^{k_{r}}+\sum_{k<k_{r}} \bar{p}_{r, k}\left(e^{-x}\right) x^{k}\right\} e^{\lambda_{r} x},
$$


with non-zero constants $c_{r, k_{r}}$. Pursuing the process, we can eliminate all exponentials $e^{\lambda_{s} x}$, except one, producing an element from the kernel of the form (3.2). This concludes the proof of the lemma.

THEOREM 3.3. The Darboux transform defined by (2.1) and (2.2) is trigonometric if and only if

$$
\operatorname{ker} P=\bigoplus_{r=1}^{n} K_{r}
$$

with $K_{r}$ a subspace of $W_{r}$ having a basis which is a union of sets of the form

$$
\left.\frac{1}{l !} \partial_{y}^{l}\left(\sum_{j=0}^{n_{r}} \sum_{k=0}^{m_{r, j}-1} c_{r, k, j} y^{k} e^{\left(\lambda_{r}-j\right) x}\right)\right|_{y=x}, \quad l=0,1, \ldots, l_{0},
$$

where $l_{0}=\max \left\{k: c_{r, k, j} \neq 0\right.$ for some $\left.j\right\}$.

Proof $^{1}$. Let us assume that the Darboux transform is trigonometric. From Lemma 3.2, we can choose a basis $\left\{\phi_{1}, \ldots, \phi_{K}\right\}$ of the kernel of $P$, such that each $\phi_{i}$ belongs to some $W_{r_{i}}, r_{i} \in\{1, \ldots, n\}$. Any $\phi \in W_{r}$ can be expanded as

$$
\phi(x)=\sum_{j=0}^{n_{r}} \sum_{k=0}^{m_{r_{r}-1}} c_{r, k, j} x^{k} e^{\left(\lambda_{r}-j\right) x} .
$$

Since the coefficients of $P$ are rational functions of $e^{x}$, they are $2 \pi i$ periodic, hence, for every $l \in \mathbb{Z}$, we have

$$
e^{-2 \pi i l \lambda_{r}} \phi(x+2 \pi i l)=\sum_{j=0}^{n_{r}} \sum_{k=0}^{m_{r-1}-1} c_{r, k, j}(x+2 \pi i l)^{k} e^{\left(\lambda_{r}-j\right) x} \in \operatorname{ker} P .
$$

Obviously, (3.4) must also hold for every $l \in \mathbb{C}$. Differentiating repetitively this identity with respect to $l$ and putting $l=0$ shows that the functions defined in (3.3) belong to the kernel of $P$ too.

Conversely, if we can choose a basis $\phi_{1}, \ldots, \phi_{K}$ of the kernel of $P$ with $\phi_{i} \in W_{r_{i}}$, then from the ith column of the determinants in the numerator and the denominator of (2.7), we can factor off $e^{\lambda_{r_{i}} x}$, which shows that the coefficients of the operator $P$ depend rationally on $x$ and $e^{x}$, i.e. $P \in \mathbb{C}\left(x, e^{x}\right)[\partial]$. From (3.3), it follows that $\phi_{1}(x+2 \pi i), \ldots, \phi_{K}(x+2 \pi i)$ is again a basis of the kernel of $P$. This shows that the coefficients of $P$ are $2 \pi i$ periodic, hence, they must be purely rational functions of $e^{x}$. The coefficients of the operator $Q$ such that $Q P=h(\partial)$ are then automatically rational functions of $e^{x}$ too, thus, completing the proof.

To specify completely the trigonometric Darboux transformation given a factorization of (3.1) as in (2.3), with $P, Q \in \mathbb{C}\left(e^{x}\right)$ [2], we still need to determine the polynomials $f(z)$ and $g(z)$ in (2.1) and (2.2), for which there is some arbitrariness. We

\footnotetext{
${ }^{1}$ The arguments used in the proof are analogous to Lemmas 2.8 and 2.9 in [2], see also [3] where conditions similar to (3.3) can be found, without proof.
} 
fix the arbitrariness by observing that there is a unique choice for the polynomial $f(z)$ such that

$$
\lim _{e^{x} \rightarrow \infty} \psi(x, z) e^{-x z}=1
$$

By an argument similar to the one given in [10] (see Lemma 6.1 in [10], where this condition is imposed when $\psi(x, z)$ is a rational function of $x$ instead of $e^{x}$ ), one can show that by normalizing the basis $\phi_{1}, \ldots, \phi_{K}$ of the kernel of $P$, as described in Theorem 3.3, so that $\phi_{i} \in W_{r_{i}}$ and

$$
\phi_{i}(x)=x^{k_{i}} e^{\left(\lambda_{r_{i}}-j_{i}\right) x}+\left(\text { terms involving only } x^{k} e^{\left(\lambda_{r i}-j\right) x} \text { with } j>j_{i}\right),
$$

one must pick

$$
f(z)=\prod_{i=1}^{K}\left(z-\lambda_{r_{i}}+j_{i}\right)
$$

Definition 3.4. The trigonometric Grassmannian $G r^{\text {trig }} \subset G r^{r a t}$ is defined to be the set of spaces $V \in G r^{r a t}$ whose stationary wave function $\psi_{V}(x, z)$ is obtained by a trigonometric Darboux transform of $e^{x z}$, with the normalization of $f(z)$ specified as in (3.6).

4. Bispectrality and Calogero-Moser matrices. Let $T$ be the shift operator acting on functions of $z$ by

$$
T f(z)=f(z+1)
$$

The pair of equations

$$
\begin{gathered}
\partial e^{x z}=z e^{x z} \\
T e^{x z}=e^{x} e^{x z}
\end{gathered}
$$

defines an anti-isomorphism between the algebra of differential operators whose coefficients are polynomials in $e^{x}$ and the algebra of (positive) difference operators whose coefficients are polynomials in $z$

$$
b: \mathbb{C}\left[e^{x}\right][\partial] \rightarrow \mathbb{C}[z][T],
$$

with

$$
b\left(e^{x}\right)=T \quad \text { and } \quad b(\partial)=z .
$$

If $\psi(x, z)$ is a trigonometric Darboux transform of $e^{x z}$, we can write the operators $P$ and $Q$ in (2.1) and (2.2) as

$$
P=\frac{1}{\theta\left(e^{x}\right)} \bar{P} \quad \text { and } \quad Q=\bar{Q} \frac{1}{v\left(e^{x}\right)} \quad \text { with } \bar{P}, \bar{Q} \in \mathbb{C}\left[e^{x}\right][\partial],
$$

and $\theta\left(e^{x}\right), v\left(e^{x}\right)$ some polynomials in $e^{x}$. 
Proposition 4.1. Let $\psi(x, z)$ be a trigonometric Darboux transform of $e^{x z}$. Then

$$
\begin{aligned}
\psi(x, z) & =\frac{1}{\theta\left(e^{x}\right)} \frac{1}{f(z)} b(\bar{P}) e^{x z} \\
e^{x z} & =\frac{1}{v\left(e^{x}\right)} b(\bar{Q}) \frac{1}{g(z)} \psi(x, z),
\end{aligned}
$$

with $\bar{P}, \bar{Q}$ as in (4.2). As a consequence, $\psi(x, z)$ in addition to be an eigenfunction of a differential operator in $x$ as in (2.4), is also an eigenfunction of a difference operator in $z$

$$
f(z)^{-1} b(\bar{P}) b(\bar{Q}) g(z)^{-1} \psi(x, z)=\theta\left(e^{x}\right) v\left(e^{x}\right) \psi(x, z),
$$

i.e. $\psi(x, z)$ solves a differential-difference bispectral problem.

Proof. Equation (4.3) follows immediately from (2.1) and (4.2), using the definition of the bispectral map (4.1). From (2.3) and (4.2), we have

$$
\bar{Q} v\left(e^{x}\right)^{-1} \theta\left(e^{x}\right)^{-1} \bar{P}=f(\partial) g(\partial),
$$

implying

$$
b(\bar{P}) \theta(T)^{-1} v(T)^{-1} b(\bar{Q})=f(z) g(z) .
$$

Hence,

$$
\psi(x, z)=f(z)^{-1} b(\bar{P}) \theta(T)^{-1} e^{x z}=g(z)\left(v(T)^{-1} b(\bar{Q})\right)^{-1} e^{x z},
$$

or, equivalently,

$$
e^{x z}=v(T)^{-1} b(\bar{Q}) g(z)^{-1} \psi(x, z) \Leftrightarrow e^{x z}=v\left(e^{x}\right)^{-1} b(\bar{Q}) g(z)^{-1} \psi(x, z),
$$

which establishes (4.4) and concludes the proof.

Let us now assume that the trigonometric Darboux transformation has been normalized as explained in (3.6), or equivalently $\psi(x, z)=\psi_{V}(x, z)$, for $V \in G r^{\text {trig }}$, according to Definition 3.4. Let us define

$$
\psi^{b}(n, z)=\psi_{V}(\log (1+z), n) .
$$

Because of the normalization, we deduce from (3.5) that

$$
\lim _{z \rightarrow \infty} \psi^{b}(n, z)(1+z)^{-n}=1
$$

Moreover, putting

$$
\Delta=T-I,
$$

after substituting $n$ for $z$ and $\log (1+z)$ for $x$ in (4.3) and (4.4), it follows that

$$
\begin{aligned}
\psi^{b}(n, z) & =\frac{1}{\theta(1+z)} R(n, \Delta)(1+z)^{n} \\
(1+z)^{n} & =\frac{1}{v(1+z)} S(n, \Delta) \psi^{b}(n, z),
\end{aligned}
$$


with $R(n, \Delta)$ and $S(n, \Delta)$ some (positive) difference operators in $\Delta$ (acting on functions depending on $n$ ), whose coefficients are rational functions of $n$. We introduce the following definition.

Definition 4.2. (i) A function $\psi(n, z)$ which satisfies (4.7) and (4.8), for some monic difference operators $R(n, \Delta), S(n, \Delta)$, with $\theta(z)$ and $v(z)$ monic polynomials in $z$ such that the order of $R$ is equal to the degree of $\theta$, will be called a discrete Darboux transform of $(1+z)^{n}$.

(ii) A discrete Darboux transform $\psi(n, z)$ of $(1+z)^{n}$, will be called polynomial when the coefficients of $R(n, \Delta)$ and $S(n, \Delta)$ are rational functions of $n$.

From Wilson's result [11] for a space $\tilde{V} \in G r^{\text {ad }}$, there exists a Calogero-Moser pair $(\tilde{X}, \tilde{Z}) \in C_{N}$ such that the corresponding tau-function is given by

$$
\tau_{\tilde{V}}\left(t_{1}, t_{2}, t_{3}, \ldots\right)=\operatorname{det}\left\{\tilde{X}-\sum_{k=1}^{\infty} k t_{k} \tilde{Z}^{k-1}\right\} .
$$

From this formula and (1.5) it follows that

$$
\tau_{\tilde{V}}\left(n, t_{1}, t_{2}, \ldots\right)=\tau_{\tilde{V}}\left(t_{1}+n, t_{2}-\frac{n}{2}, t_{3}+\frac{n}{3}, \ldots\right)
$$

is a tau-function of the discrete KP hierarchy

$$
\frac{\partial L}{\partial t_{i}}=\left[\left(L^{i}\right)_{+}, L\right], \quad L=\Delta+\sum_{j=0}^{\infty} a_{j}\left(n, t_{1}, t_{2}, \ldots\right) \Delta^{-j},
$$

with $\left(L^{i}\right)_{+}$the (positive) difference part of $L^{i}$. The corresponding wave function (of the discrete KP hierarchy) is

$$
\begin{aligned}
\psi_{\tilde{V}}(n, t, z)=(1+z)^{n} \exp ( & \left.\sum_{k=1}^{\infty} t_{k} z^{k}\right) \\
& \times \frac{\tau_{\tilde{V}}\left(n, t_{1}-\frac{1}{z}, t_{2}-\frac{1}{2 z^{2}}, t_{3}-\frac{1}{3 z^{3}}, \ldots\right)}{\tau_{\tilde{V}}\left(n, t_{1}, t_{2}, t_{3}, \ldots\right)} .
\end{aligned}
$$

A simple computation using (4.9) shows that

$$
\tau_{\tilde{V}}\left(n, t_{1}, t_{2}, \ldots\right)=\operatorname{det}\left\{\tilde{X}-\sum_{k=1}^{\infty} k t_{k} \tilde{Z}^{k-1}-n(I+\tilde{Z})^{-1}\right\} .
$$

In [6] it was assumed that the eigenvalues of $\tilde{Z}$ are inside the unit circle, but clearly the right-hand side of (4.11) is well defined as long as -1 is not an eigenvalue of $\tilde{Z}$. Thus, analytic continuation shows that the above formula can be applied when $\operatorname{det}(I+\tilde{Z}) \neq 0$.

If we denote by $\psi_{\tilde{V}}(n, z)=\psi_{\tilde{V}}(n, 0, z)$, the corresponding stationary wave function, then from (4.10) and (4.11) it follows immediately that

$$
\lim _{n \rightarrow \infty} \psi_{\tilde{V}}(n, z)(1+z)^{-n}=1
$$


It was shown in [6] that $\psi_{\tilde{V}}(n, z)$ is a polynomial discrete Darboux transform of $(1+z)^{n}$. In fact, any polynomial discrete Darboux transform of $(1+z)^{n}$ is obtained by the above construction, up to a normalization, which can be fixed by imposing (4.12). The result is summarized in the next theorem, of which we sketch the idea of the proof.

TheOREM 4.3. A function $\psi(n, z)$ is a polynomial discrete Darboux transform of $(1+z)^{n}$ if and only if

$$
\psi(n, z)=\frac{\theta_{1}(z)}{\theta_{2}(z)} \psi_{\tilde{V}}(n, z),
$$

where $\theta_{1}(z)$ and $\theta_{2}(z)$ are monic polynomials of the same degree, and $\psi_{\tilde{V}}(n, z)$ is a stationary wave function of the discrete KP hierarchy as in (4.10) and (4.11), built from a space $\tilde{V} \in G r^{\text {ad }}$ corresponding to a Calogero-Moser pair $(\tilde{X}, \tilde{Z})$ such that $\operatorname{det}(I+\tilde{Z}) \neq 0$.

The proof of the above theorem can be briefly explained as follows. First we show that, up to a factor independent of $n$, polynomial discrete Darboux transforms of $(1+z)^{n}$ can be characterized by the fact that the kernel of the operator $R$ in (4.7) has a basis consisting of functions of the form

$$
\phi_{j}(n)=p_{j}(n)\left(\lambda_{j}+1\right)^{n}, \text { where } \lambda_{j} \in \mathbb{C} \backslash\{-1\},
$$

and $p_{j}(n)$ are polynomials of $n$. The space $\tilde{V} \in G r^{a d}$ corresponds to a Darboux transform of $e^{x z}$ (in the sense of Definition 2.1) such that the polynomial $P(x, \partial)$ in (2.1) has a kernel spanned by the functions

$$
f_{j}(x)=\left.p_{j}\left((1+z) \partial_{z}\right) e^{x z}\right|_{z=\lambda_{j}}
$$

The condition $\operatorname{det}(I+\tilde{Z}) \neq 0$ in the theorem reflects the fact that $\lambda_{j} \neq-1$.

In this paper, we just like to explain how to deduce from this result a parametrization of the trigonometric Grassmannian $\mathrm{Gr}^{\text {trig }}$ in terms of trigonometric Calogero-Moser matrices as defined in (1.4). We recall that Kasman and Gekhtman (see [8], Corollary 3.2) have established that for any triplet $(X, Y, Z)$ of $N \times N$ matrices such that $\operatorname{rank}(X Z-Y X)=1$, the function

$$
\tau_{(X, Y, Z)}\left(t_{1}, t_{2}, \ldots\right)=\operatorname{det}\left\{I-X \exp \left\{-\sum_{k=1}^{\infty} t_{k} Z^{k}\right\} \exp \left\{\sum_{k=1}^{\infty} t_{k} Y^{k}\right\}\right\}
$$

is a tau-function of the KP hierarchy, corresponding to some space of $G r^{r a t}$. The next theorem shows that the special choice $Y=Z-I$ in their formula, with $X$ invertible, characterizes tau-functions of spaces of $\mathrm{Gr}^{\text {trig }}$.

THEOREM 4.4. There is a one-to-one correspondence between trigonometric Calogero-Moser pairs $(X, Z)$ (modulo conjugation) as defined in (1.4), and tau-functions of spaces $V \in G r^{\text {trig }}$, which is given by

$$
\tau_{V}\left(t_{1}, t_{2}, \ldots\right)=\operatorname{det}\left\{I-X \exp \left\{\sum_{k=1}^{\infty} t_{k}\left((Z-I)^{k}-Z^{k}\right)\right\}\right\} .
$$

Proof. Let $\psi_{V}(x, z)$ be the stationary wave function of a space $V \in G r^{\text {trig }}$. From (4.6)-(4.8), it follows that $\psi^{b}(n, z)$ as defined in (4.5) is a polynomial Darboux transform 
of $(1+z)^{n}$ such that

$$
\lim _{n \rightarrow \infty} \psi^{b}(n, z)(1+z)^{-n}=1
$$

From Theorem 4.3 and (4.12), there exists a space $\tilde{V} \in G r^{a d}$ and a pair of matrices $(\tilde{X}, \tilde{Z})$ such that $\psi^{b}(n, z)=\psi_{\tilde{V}}(n, z)$ can be computed via formulae (4.10) and (4.11). By an easy computation we obtain

$$
\psi^{b}(n, z)=(1+z)^{n} \operatorname{det}\left\{I+\left(\tilde{X}-n(I+\tilde{Z})^{-1}\right)^{-1}(z I-\tilde{Z})^{-1}\right\} .
$$

Hence, by the definition of $\psi^{b}(n, z)$ in (4.5),

$$
\begin{aligned}
\psi_{V}(x, z) & =\psi^{b}\left(z, e^{x}-1\right) \\
& =e^{x z} \operatorname{det}\left\{I+\left(\tilde{X}-z(I+\tilde{Z})^{-1}\right)^{-1}\left(\left(e^{x}-1\right) I-\tilde{Z}\right)^{-1}\right\} .
\end{aligned}
$$

Defining

$$
X=I+\tilde{Z}^{t}, \quad Z=\tilde{X}^{t}\left(I+\tilde{Z}^{t}\right),
$$

since a determinant is invariant by transposition, we obtain

$$
\begin{aligned}
\psi_{V}(x, z) & =e^{x z} \operatorname{det}\left\{I+\left(e^{x} I-\left(I+\tilde{Z}^{t}\right)\right)^{-1}\left(\tilde{X}^{t}-z\left(I+\tilde{Z}^{t}\right)^{-1}\right)^{-1}\right\} \\
& =e^{x z} \operatorname{det}\left\{I-X\left(e^{x} I-X\right)^{-1}(z I-Z)^{-1}\right\},
\end{aligned}
$$

where in the last equation, we have used that the multiplication of the matrices $X$ and $\left(e^{x} I-X\right)^{-1}$ commutes. Since $(\tilde{X}, \tilde{Z})$ is a Calogero-Moser pair, we deduce that

$$
\begin{aligned}
\operatorname{rank}\left(X Z X^{-1}-Z+I\right) & =\operatorname{rank}\left(\left[X, Z X^{-1}\right]+I\right) \\
& =\operatorname{rank}\left(\left[\tilde{Z}^{t}, \tilde{X}^{t}\right]+I\right)=\operatorname{rank}([\tilde{X}, \tilde{Z}]+I)=1,
\end{aligned}
$$

showing that $(X, Z)$ in (4.14) is a trigonometric Calogero-Moser pair.

On the other hand, denoting for short by $\exp \{\ldots\}$ the expression that appears inside the exponential in (4.13), one computes

$$
\begin{aligned}
& \tau_{V}\left(t_{1}-\frac{1}{z}, t_{2}-\frac{1}{2 z^{2}}, t_{3}-\frac{1}{3 z^{3}}, \ldots\right) \\
& =\operatorname{det}\left\{I-X \exp \{\ldots\}(z I-(Z-I))(z I-Z)^{-1}\right\} \\
& =\operatorname{det}\left\{I-X \exp \{\ldots\}-X \exp \{\ldots\}(z I-Z)^{-1}\right\},
\end{aligned}
$$

from which it follows that

$$
\begin{aligned}
& \frac{\tau_{V}\left(t_{1}-\frac{1}{z}, t_{2}-\frac{1}{2 z^{2}}, t_{3}-\frac{1}{3 z^{3}}, \ldots\right)}{\tau_{V}\left(t_{1}, t_{2}, t_{3}, \ldots\right)} \\
& =\operatorname{det}\left\{I-X\left(\exp ^{-1}\{\ldots\}-X\right)^{-1}(z I-Z)^{-1}\right\} .
\end{aligned}
$$

Putting $t_{1}=x, t_{2}=t_{3}=\cdots=0$ in this formula shows that $\psi_{V}(x, z)$ in (4.15) satisfies Sato's formula, with $\tau_{V}$ as in (4.13). Since this formula determines the tau-function up to a constant, the proof is complete. 
ACKnOwledgements. The research of Luc Haine is supported by the Belgian Interuniversity Attraction Pole P06/02 and the European Science Foundation Program MISGAM. Emil Horozov acknowledges the support by grant MI 1504/2005 of the National Fund 'Scientific research' of the Bulgarian Ministry of Education and Science.

\section{REFERENCES}

1. B. Bakalov, E. Horozov and M. Yakimov, Bäcklund-Darboux transformations in Sato's Grassmannian, Serdica Math. J. 22(4) (1996), 571-588.

2. B. Bakalov, E. Horozov and M. Yakimov, Bispectral algebras of commuting ordinary differential operators, Commun. Math. Phys. 190 (1997), 331-373.

3. O. A. Chalykh and F. W. Nijhoff, Bispectral rings of difference operators, Russ. Math. Surv. 54(3) (1999), 644-645.

4. L. A. Dickey, Soliton equations and Hamiltonian systems, Second Edition, Advanced Series in Mathematical Physics 26 (World Scientific Publishing Co., River Edge, NJ, 2003).

5. L. Haine, KP trigonometric solitons and an adelic flag manifold, SIGMA Symm. Integr. Geom. Meth. Appl. 3 (Paper 015), (2007), 15 pages.

6. L. Haine and P. Iliev, Commutative rings of difference operators and an adelic flag manifold, Int. Math. Res. Not. 2000(6) (2000), 281-323.

7. P. Iliev, Rational Ruijsenaars-Schneider hierarchy and bispectral difference operators, Phys. D 229(2) (2007), 184-190.

8. A. Kasman and M. Gekhtman, Solitons and almost-intertwining matrices, J. Math. Phys. 42 (2001), 3540-3551.

9. E. Mukhin, V. Tarasov and A. Varchenko, Bispectral and $\left(g l_{N}, g l_{M}\right)$ dualities, discrete versus differential, Adv. Math. 218(1) (2008), 216-265.

10. G. Wilson, Bispectral commutative ordinary differential operators, J. Reine Angew. Math. 442 (1993), 177-204.

11. G. Wilson, Collisions of Calogero-Moser particles and an adelic Grassmannian, Invent. Math. 133 (1998), 1-41. 